\title{
An Anthropomorphic Phantom Study of Brain Dopamine Transporter SPECT Images Obtained Using Different SPECT/CT Devices and Collimators
}

\author{
Akira Maebatake ${ }^{1}$, Maho $\mathrm{Sato}^{2}$, Ruriko Kagami ${ }^{2}$, Yasuo Yamashita $^{3}$, Isao Komiya ${ }^{3}$, Kazuhiko Himuro ${ }^{3}$, \\ Shingo $\mathrm{Baba}^{4}$, and Masayuki Sasaki ${ }^{1}$ \\ ${ }^{I}$ Division of Medical Quantum Science, Department of Health Sciences, Graduate School of Medical Sciences, Kyushu University, \\ Fukuoka, Japan; ${ }^{2}$ Radiological Science Course, Department of Health Sciences, School of Medicine, Kyushu University, Fukuoka, \\ Japan; ${ }^{3}$ Division of Radiology, Department of Medical Technology, Kyushu University Hospital, Fukuoka, Japan; and ${ }^{4}$ Department \\ of Clinical Radiology, Graduate School of Medical Sciences, Kyushu University, Fukuoka, Japan
}

The aim of this study was to evaluate differences in dopamine transporter SPECT images among different SPECT/CT devices and to determine the most appropriate region of interest (ROI) for semiquantitative evaluation. Methods: An anthropomorphic striatal phantom was filled with ${ }^{123}$ I solutions of different striatumto-background radioactivity ratios. Data were acquired using 2 SPECT/CT devices equipped with low- to medium-energy generalpurpose and low-energy high-resolution (LEHR) collimators. The SPECT images were reconstructed by filtered backprojection with both attenuation and scatter correction and then were analyzed using specific binding ratio (SBR). The most appropriate of $7 \mathrm{ROI}$ types was determined, and we then compared the linearity and recovery of SBR among the different SPECT/CT devices and collimators. Results: The linearity of SBR was excellent for all types of ROls. The ROI contouring the striatum based on the CT images showed the best recovery of SBR using mean activity in the striatal $\mathrm{ROI}\left(\mathrm{SBR}_{\text {mean }}\right)(47.8 \%)$. For this ROI, the recovery of SBR mean for SPECT/CT with a LEHR collimator with thick septa and a long hole length was $61.6 \%$ significantly higher than that of other devices. Conclusion: The $\mathrm{ROI}$ contouring the striatum based on CT images was considered appropriate for evaluating dopamine transporter SPECT/CT. Among the different SPECT/CT devices, an LEHR collimator designed for ${ }^{123} \mid$ imaging is recommended.

Key Words: SPECT/CT; dopamine transporter; region of interest

J Nucl Med Technol 2015; 43:41-46

DOI: 10.2967/jnmt.114.149401

I maging of the dopamine transporter (DaT) using radiopharmaceuticals that bind to it is an important tool for diagnosing neurologic disorders $(1,2)$. Some movement disorders

Received Oct. 1, 2014; revision accepted Oct. 30, 2014.

For correspondence contact: Masayuki Sasaki, Division of Medical Quantum Science, Department of Health Sciences, Graduate School of Medical Sciences, Kyushu University, 3-1-1 Maidashi, Higashi-ku, Fukuoka 812-8582, Japan.

E-mail: msasaki@hs.med.kyushu-u.ac.jp

Published online Dec. 23, 2014.

COPYRIGHT (c) 2015 by the Society of Nuclear Medicine and Molecular Imaging, Inc. are associated with a loss of dopaminergic nerve terminals in the nigrostriatal pathway. Especially in the early phase of Parkinson disease, differentiation from essential tremor, vascular parkinsonism, and multiple-system atrophy is sometimes difficult (3-6).

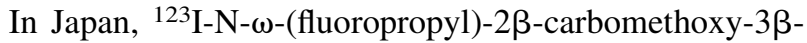
(4-iodophenyl)tropane ( ${ }^{123} \mathrm{I}$-FP-CIT) became available for routine clinical use in January 2014. Many studies examining ${ }^{123}$ I-FP-CIT SPECT imaging of DaT for diagnosing Parkinson disease have been reported (7-9). In combination with semiquantification, SPECT imaging of DaT has been reported to improve diagnostic accuracy for some movement disorders and to be useful for monitoring disease progression (10-15).

Recently, guidelines to obtain standard DaT SPECT images have been published $(1,2)$. However, different images are considered to be obtained when different devices and collimators are used, as has been reported for cerebral perfusion SPECT (16). Therefore, it is important to evaluate differences in DaT SPECT images among different devices and collimators. Furthermore, semiquantitative indices for DaT SPECT, such as the specific binding ratio (SBR), are widely used for diagnosis. Determination of the region of interest (ROI) on the striatum and reference regions is therefore also important to standardize the semiquantitative evaluation of DaT SPECT imaging. The examination protocol for ${ }^{123} \mathrm{I}-$ FP-CIT SPECT should be harmonized to obtain standardized results among different devices and different institutions.

The aim of this study was to evaluate differences in DaT SPECT images among different devices and to determine the most appropriate ROI for evaluating SBR on SPECT/CT.

\section{MATERIALS AND METHODS}

\section{Phantoms}

A pool phantom with a $16-\mathrm{cm}$ diameter and $15-\mathrm{cm}$ height (Akita Machine-Engineering) was used to determine the attenuation coefficient using the Chang method (17). The phantom was filled with a $16.8 \mathrm{kBq} / \mathrm{mL}$ solution of ${ }^{123} \mathrm{I}$.

A 3-dimensional (3D) brain phantom (Molecular Imaging Labo Inc.) was used to determine the parameters for a Butterworth filter 
(Fig. 1A) (18). The 3D brain phantom was made of a transparent photocurable polymer, or polyepoxide, with a density of $1.07 \mathrm{~g} / \mathrm{mL}$ and was constructed with a laser-modeling technique. It was a structure precisely imitating the gray matter, white matter, cerebrospinal fluid space, skull, and scalp based on MR images. The bone region was filled with $\mathrm{K}_{2} \mathrm{HPO}_{4}(310.3 \mathrm{~mL})$. The gray matter region was filled with a $25 \mathrm{kBq} / \mathrm{mL}$ solution of ${ }^{123} \mathrm{I}$.

An anthropomorphic striatal phantom (NMP Business Support Co. Ltd.) was used to evaluate DaT SPECT images (Fig. 1B). It consisted of chambers for the right and left striatum $(12.5 \mathrm{~mL})$ and for the cerebrum $(1,180 \mathrm{~mL})$ based on MR images of a healthy subject. The chambers were surrounded by structures simulating skin and skull. The striatum and background were filled with different ${ }^{123}$ I solutions (Table 1). Four striatum-to-background radioactivity ratios $(\mathrm{S} / \mathrm{B}$ ratios) $(8.08,6.03,4.03$, and 3.01$)$ were examined.

\section{Imaging Protocol}

The SPECT/CT data were acquired using 2 different devices: a Symbia T6 (Siemens Healthcare) equipped with a low- to mediumenergy general-purpose collimator (camera 1) or a low-energy highresolution (LEHR) collimator (camera 2) and an Infinia Hawkeye 4 (GE Healthcare) equipped with an extended low-energy generalpurpose collimator (camera 3) or an LEHR collimator (camera 4). The main energy window was $159 \mathrm{keV} \pm 10 \%$ width. The subwindows with $7 \%$ width were set just above and below the main window for scatter correction. The matrix size was $128 \times 128$, and the pixel size was $3.3 \mathrm{~mm}$ (1.45 zoom for Symbia T6 and 1.34 zoom for Infinia Hawkeye 4). The data were acquired 6 times in continuous mode with clockwise and counterclockwise rotation

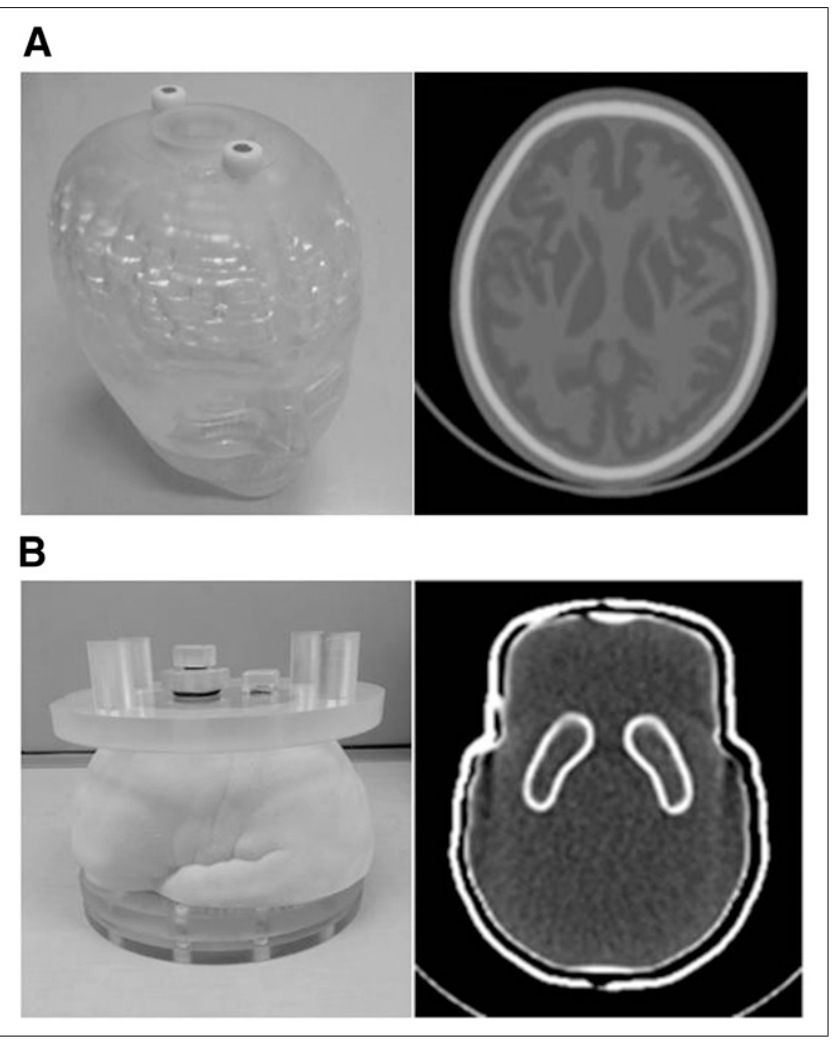

FIGURE 1. (A) Appearance and CT image of 3D brain phantom. (B) Appearance and CT image of anthropomorphic striatal phantom.
TABLE 1

Radioactivity of Right and Left Striatum and Background in Striatal Phantom

\begin{tabular}{cccc}
\hline & \multicolumn{2}{c}{ Striatum } & \\
\cline { 2 - 3 } Experiment & Right & Left & Background \\
\hline Experiment 1 & $40.4 \mathrm{kBq} / \mathrm{mL}$ & $20.2 \mathrm{kBq} / \mathrm{mL}$ & $5.0 \mathrm{kBq} / \mathrm{mL}$ \\
S/B ratio & 8.08 & 4.03 & \\
SBR & 7.08 & 3.03 & \\
Experiment 2 & $40.4 \mathrm{kBq} / \mathrm{mL}$ & $20.2 \mathrm{kBq} / \mathrm{mL}$ & $6.7 \mathrm{kBq} / \mathrm{mL}$ \\
S/B ratio & 6.03 & 3.01 & \\
SBR $_{\text {true }}$ & 5.03 & 2.01 & \\
\hline
\end{tabular}

for $5 \mathrm{~min}$ per $180^{\circ}$. Attenuation was corrected using the Chang method. The triple-energy window method was used for scatter correction. Images were reconstructed on a workstation using Syngo MI applications (Siemens Healthcare) and a Xeleris 2 device (GE Healthcare).

\section{Reconstruction Parameters}

The SPECT images were reconstructed using filtered backprojection with a ramp filter. Before reconstruction, the data were processed using a Butterworth filter.

The attenuation coefficient for the Chang method was determined by a pool phantom analysis (19). At that time, a Butterworth filter was used for smoothing (order, 8; cutoff frequency, 0.55 cycles/cm for the Symbia T6 and 0.50 cycles/cm for the Infinia Hawkeye 4). The attenuation coefficient varied from 0.06 to 0.14 . The pool phantom image was visually evaluated for flatness of the profile curve, and the coefficient of variance (CV) and summed difference from reference activity were determined. Five nuclear medicine physicians classified the flatness of the profile curve as -2 for obviously concave, -1 for probably concave, 0 for flat, +1 for probably convex, or +2 for obviously convex. The CV was calculated by dividing the percentage SD by the mean activity of a 16-cm-diameter circular ROI on the phantom image. The summed difference from the reference activity was calculated as follows: A $30 \times 35$ pixel rectangular ROI was placed on the reconstructed image. The mean activity of the 2 top-corner pixels was used as a reference value. The difference between the reference activity and the value of each pixel in the ROI was summed (positive: convex; negative: concave). The $\mu$ values were finally determined by evaluating these results comprehensively.

The cutoff frequency of the Butterworth filter, which was determined by evaluating the SPECT images of the 3D brain phantom, varied from 0.20 to 0.70 by increments of 0.02 . At that time, the attenuation coefficient determined by the above study and the order for the Butterworth filter (with an order of 8) were used. The 5 nuclear medicine physicians visually graded the $3 \mathrm{D}$ brain phantom image as -2 for extremely poor, -1 for poor, 0 for normal, +1 for good, or +2 for excellent.

\section{SBR}

SBR was obtained by subtracting the mean counts of the background area $\left(\mathrm{C}_{\mathrm{b}}\right)$ from the counts of the striatum $\left(\mathrm{C}_{\mathrm{s}}\right)$ and $\mathrm{C}_{\mathrm{b}}(1,2)$ :

$$
\mathrm{SBR}=\frac{\mathrm{C}_{\mathrm{s}}-\mathrm{C}_{\mathrm{b}}}{\mathrm{C}_{\mathrm{b}}}
$$

SBR using the mean SPECT count of the striatum $\left(\mathrm{C}_{\mathrm{s}}\right.$, mean $)$ is referred to as $\mathrm{SBR}_{\text {mean }}$, whereas SBR using the maximum SPECT 
TABLE 2

ROIs of DaT SPECT Images

\begin{tabular}{|c|c|}
\hline $\mathrm{ROI}$ & Determination \\
\hline $\mathrm{ROI}_{\max }$ & Maximum count in striatum \\
\hline $\mathrm{ROI}_{\mathrm{CT} 2}, \mathrm{ROI}_{\mathrm{CT} 3}$ & $\begin{array}{l}\text { Contour of striatum determined } \\
\text { by CT image }\end{array}$ \\
\hline $\mathrm{ROI}_{\mathrm{SP} 2}, \mathrm{ROI}_{\mathrm{SP} 3}$ & $\begin{array}{l}\text { Contour of striatum determined by } \\
\text { SPECT image }\end{array}$ \\
\hline $\mathrm{ROI}_{\mathrm{B} \times 2}, \mathrm{ROI}_{\mathrm{B} \times 3}$ & $15 \times 12$ pixel rectangle \\
\hline Background & $10 \times 10$ pixel rectangle on occipital lobe \\
\hline
\end{tabular}

count of the striatum $\left(\mathrm{C}_{\mathrm{s}, \max }\right)$ is referred to as $\mathrm{SBR}_{\max }$. $\mathrm{SBR}$ on SPECT images $\left(\mathrm{SBR}_{\mathrm{SPECT}}\right)$ consisted of both $\mathrm{SBR}_{\text {mean }}$ and $\mathrm{SBR}_{\max }$. True SBR ( $\left.\mathrm{SBR}_{\text {true }}\right)$ was calculated using the radioactivity in the striatum and the background as measured by an automatic well $\gamma$ counter (AccuFLEX $\gamma 7001$; Hitachi Aloka Medical, Ltd.) and was used as a reference value (Table 1). S/B was 8.08, $6.03,4.03$, and 3.01, respectively, and $\mathrm{SBR}_{\text {true }}$ was 7.08, 5.03, 3.03 , and 2.01, respectively.

\section{ROls}

Seven types of ROI were compared to determine the most appropriate one for evaluating SBR on SPECT images (Table 2). $\mathrm{ROI}_{\text {max }}$ extracted the highest count of the striatum. ROI $\mathrm{CT}_{2}$ was the contour of each striatum determined by the CT image (Fig. 2A) and was placed on the slice with the maximum count for the striatum (Fig. 2B). $\mathrm{ROI}_{\mathrm{CT} 3}$ was the same shape as $\mathrm{ROI}_{\mathrm{CT} 2}$ but was placed on 5 slices for averaging. $\mathrm{ROI}_{\mathrm{SP} 2}$ was equal to the contour of each striatum determined by SPECT imaging and was placed on the slice with the maximum count for the striatum (Fig. 2C). $\mathrm{ROI}_{\mathrm{SP} 3}$ was the same shape as $\mathrm{ROI}_{\mathrm{SP} 2}$ but was placed on 5 slices for averaging. $\mathrm{ROI}_{\mathrm{BX} 2}$ was rectangular, with $15 \times 12$ pixels, including the whole striatum on the slice with the maximum count (Fig. 2D). $\mathrm{ROI}_{\mathrm{BX} 3}$ was equal to $\mathrm{ROI}_{\mathrm{BX} 2}$ but was placed on 5 slices for averaging. In all ROIs except $\mathrm{ROI}_{\max }$, the count in the striatum was averaged $\left(\mathrm{C}_{\mathrm{s}}\right.$, mean $)$. The ROI for the background, within a $10 \times$ 10 pixel rectangle, was placed on the occipital region. These ROIs were placed manually and individually by a nuclear medicine physician and 2 radiologic technologists.

\section{Data Analysis}

$\mathrm{SBR}_{\text {SPECT }}\left(\mathrm{SBR}_{\text {mean }}\right.$ and $\left.\mathrm{SBR}_{\text {max }}\right)$ data were compared by examining recovery and linearity in a comparison with the true radioactivity. Recovery was the percentage of $\mathrm{SBR}_{\mathrm{SPECT}}$ to $\mathrm{SBR}_{\text {true }}$,

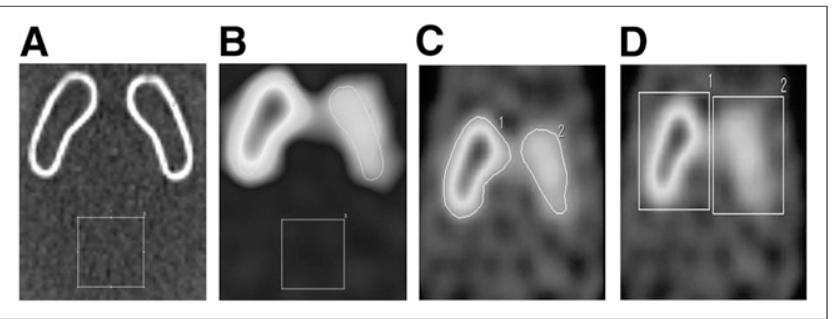

FIGURE 2. Striatal ROls: contour of striatum and background determined by $\mathrm{CT}$ images $\left(\mathrm{ROI}_{\mathrm{CT} 2}\right)(\mathrm{A}), \mathrm{ROI}_{\mathrm{CT} 2}$ overlaid on SPECT image $(B), \mathrm{ROI}_{\mathrm{SP} 2}$ determined by contouring striatum on SPECT image (C), and box ROls, including whole striatum $\left(\mathrm{ROI}_{\mathrm{B} \times 2}\right)(\mathrm{D}) .1$ = right striatal $\mathrm{ROI} ; 2=$ left striatal $\mathrm{ROI} ; 3=$ background ROI.
TABLE 3

Reconstruction Parameters of Each Device

\begin{tabular}{cccc}
\hline & $\begin{array}{c}\mu \text { value } \\
\text { of Chang } \\
\text { with collimator }\end{array}$ & \multicolumn{2}{c}{$\begin{array}{c}\text { Butterworth filter } \\
\text { (cycles/cm) }\end{array}$} \\
\cline { 3 - 4 } method $\left(\mathrm{cm}^{-1}\right)$ & Order & Cutoff \\
\hline Camera 1 & 0.12 & 8 & 0.40 \\
Camera 2 & 0.08 & 8 & 0.36 \\
Camera 3 & 0.12 & 8 & 0.44 \\
Camera 4 & 0.12 & 8 & 0.44
\end{tabular}

and the difference was analyzed by the Tukey-Kramer test. Linearity was analyzed by determining the correlation coefficient between $\mathrm{SBR}_{\mathrm{SPECT}}$ and $\mathrm{SBR}_{\text {true. }}$ A $P$ value of less than 0.05 was considered significant. The reproducibility of $\mathrm{SBR}_{\mathrm{SPECT}}$ was evaluated using the intraclass correlation coefficient (ICC) and CV. The reproducibility for each ROI determination was classified according to the ICC value (0-0.20, slight; 0.21-0.40, fair; 0.41-0.60, moderate; 0.61-0.80, substantial; 0.81-1.00, almost perfect). The CV was calculated using the mean count and SD. The most appropriate ROI was determined using images obtained by a Symbia T6 equipped with a low- to medium-energy generalpurpose collimator.

\section{RESULTS}

\section{Determination of Reconstruction Parameters}

The reconstruction parameters for each device and collimator were determined as listed in Table 3. The $\mu$ value for Chang attenuation correction was $0.12 \mathrm{~cm}^{-1}$ except for camera 2, which was $0.08 \mathrm{~cm}^{-1}$. The cutoff frequency of the Butterworth filter for each device ranged from 0.36 to 0.44 . Figure 3 shows the reconstructed SPECT images. The variability of the background in cameras 2 and 4 was inferior to that in cameras 1 and 3 . On the other hand, striatal uptake appeared broad and rounded on the images from cameras 1 and 3 .

\section{Assessment of ROls}

The recovery and linearity of $\mathrm{SBR}_{\mathrm{SPECT}}$ using the 7 ROIs were compared with those for $\mathrm{SBR}_{\text {true }}$ (Table 4). Recovery of $\mathrm{SBR}_{\text {SPECT }}$ was best for $\mathrm{ROI}_{\max }(75.3 \%)$ and was significantly higher than for any of the other ROIs $(P<0.05)$. ROI $_{\mathrm{CT} 2}$ had the second highest value $(47.8 \%)$ and was also significantly higher than any of the other ROIs, except ROI $\mathrm{Rax}_{\text {. }}$ SBR $_{\text {SPECT }}$ showed good linearity for all ROIs $\left(R^{2}>0.95\right)$.

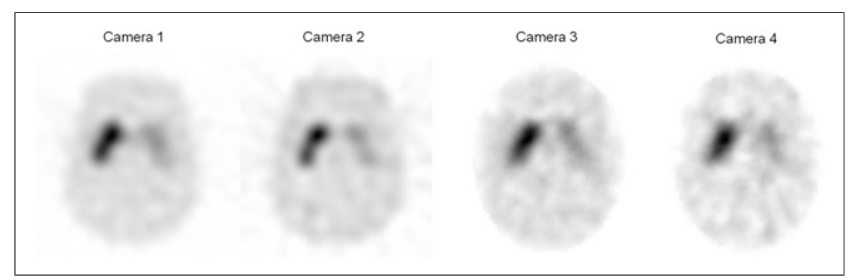

FIGURE 3. Reconstructed SPECT images of anthropomorphic striatal phantom obtained using different devices and collimators. 
TABLE 4

Recovery and Linearity of SBR SPECT $_{\text {Calculated for } 7 \text { ROIs }}$

\begin{tabular}{|c|c|c|c|c|c|c|}
\hline \multirow[b]{2}{*}{$\mathrm{ROI}$} & \multicolumn{4}{|c|}{$\mathrm{SBR}_{\text {true }}$} & \multirow[b]{2}{*}{ Average } & \multirow[b]{2}{*}{ Linearity $R^{2}$} \\
\hline & 2.01 & 3.03 & 5.03 & 7.08 & & \\
\hline $\mathrm{ROI}_{\max }$ & $87.1 \%$ & $67.1 \%$ & $74.5 \%$ & $72.4 \%$ & $75.3 \% \pm 7.3 \%^{*}$ & 0.95 \\
\hline $\mathrm{ROI}_{\mathrm{CT} 2}$ & $50.0 \%$ & $47.5 \%$ & $46.8 \%$ & $47.1 \%$ & $47.8 \% \pm 1.3 \%^{\dagger}$ & 0.96 \\
\hline $\mathrm{ROI}_{\text {CT3 }}$ & $49.0 \%$ & $45.9 \%$ & $45.8 \%$ & $46.1 \%$ & $46.7 \% \pm 1.3 \%$ & 0.96 \\
\hline $\mathrm{ROI}_{\mathrm{SP} 2}$ & $47.6 \%$ & $43.0 \%$ & $32.1 \%$ & $32.9 \%$ & $38.9 \% \pm 6.6 \%$ & 0.97 \\
\hline $\mathrm{ROI}_{\mathrm{SP} 3}$ & $44.1 \%$ & $42.2 \%$ & $29.4 \%$ & $31.3 \%$ & $36.7 \% \pm 6.5 \%$ & 0.95 \\
\hline $\mathrm{ROI}_{\mathrm{BX} 2}$ & $25.2 \%$ & $21.8 \%$ & $20.1 \%$ & $19.3 \%$ & $21.6 \% \pm 2.3 \%$ & 0.99 \\
\hline $\mathrm{ROI}_{\mathrm{B} \times 3}$ & $22.5 \%$ & $19.2 \%$ & $18.2 \%$ & $17.2 \%$ & $19.3 \% \pm 2.0 \%$ & 0.99 \\
\hline $\begin{array}{l}{ }^{*} P<0.05 . \\
{ }^{\dagger} P<0.05 .\end{array}$ & & & & & & \\
\hline
\end{tabular}

The reproducibility of $\mathrm{SBR}_{\mathrm{SPECT}}$ in the 7 ROIs is shown in Table 5. ICC agreed well for all ROIs $(>0.80)$. The CVs of $\mathrm{ROI}_{\mathrm{max}}, \mathrm{ROI}_{\mathrm{CT} 2}, \mathrm{ROI}_{\mathrm{CT} 3}, \mathrm{ROI}_{\mathrm{SP} 2}$, and $\mathrm{ROI}_{\mathrm{SP} 3}$ were lower than $5 \%$, whereas those of the box ROIs $\left(\mathrm{ROI}_{\mathrm{BX} 2}\right.$ and $\left.\mathrm{ROI}_{\mathrm{BX} 3}\right)$ were higher than the other ROIs. The best CV was observed for $\mathrm{ROI}_{\max }(1.75 \%)$. On the basis of these results, the subsequent analyses were performed using $\mathrm{ROI}_{\max }$ and $\mathrm{ROI}_{\mathrm{CT} 2}$.

\section{Comparison of Different Devices and Collimators}

The recovery of SBR $_{\text {SPECT }}$ was compared among the different SPECT devices and collimators (Fig. 4; Table 6). The best recovery was for $\mathrm{SBR}_{\text {mean }}(61.6 \%)$ and $\mathrm{SBR}_{\max }$ $(92.9 \%)$ for camera 4 . This result was significantly higher than for the other devices $(P<0.05)$. Although the recoveries for the other devices did not show any significant differences, the lowest recovery was for $\mathrm{SBR}_{\text {mean }}$ and $\mathrm{SBR}_{\max }(41.0 \%$ and $59.6 \%$, respectively) for camera 2 . Considering the linearity of $\mathrm{SBR}_{\mathrm{SPECT}}$, all cameras showed good linearity for both $\mathrm{SBR}_{\max }$ and $\mathrm{SBR}_{\text {mean }}\left(R^{2}>0.90\right)$.

\section{DISCUSSION}

In this study, we examined differences in DaT SPECT images of an anthropomorphic striatal phantom obtained using different devices and collimators, and we determined the most appropriate ROI for quantitative analysis. The best result was obtained using $\mathrm{ROI}_{\max }$, followed by $\mathrm{ROI}_{\mathrm{CT} 2}$. In the comparison of different devices and collimators, camera 4 gave the best results, followed by camera 3 .

TABLE 5

Reproducibility of SBR SPECT $_{\text {Calculated by the } 7 \text { ROls }}$

\begin{tabular}{cccc}
\hline $\mathrm{ROI}$ & $\mathrm{ICC}$ & $95 \% \mathrm{Cl}$ & $\mathrm{CV}$ \\
\hline $\mathrm{ROI}_{\max }$ & 0.994 & $0.935-1.000$ & $1.75 \%$ \\
$\mathrm{ROI}_{\mathrm{CT} 2}$ & 0.985 & $0.881-0.999$ & $2.64 \%$ \\
$\mathrm{ROI}_{\mathrm{CT} 3}$ & 0.981 & $0.846-0.999$ & $2.59 \%$ \\
$\mathrm{ROI}_{\mathrm{SP} 2}$ & 0.983 & $0.912-0.999$ & $2.58 \%$ \\
$\mathrm{ROI}_{\mathrm{SP3}}$ & 0.982 & $0.876-0.999$ & $1.83 \%$ \\
$\mathrm{ROI}_{\mathrm{BX} 2}$ & 0.955 & $0.686-0.997$ & $8.34 \%$ \\
$\mathrm{ROI}_{\mathrm{BX} 3}$ & 0.980 & $0.842-0.999$ & $4.60 \%$ \\
\hline
\end{tabular}

Our results showed that the best recovery and reproducibility of SBR was obtained using $\mathrm{ROI}_{\max }$, followed by $\mathrm{ROI}_{\mathrm{CT} 2}$. In some patients with Parkinson disease, ${ }^{123}$ I-FP-CIT uptake in the striatum has been reported to show a heterogeneous distribution (20). Additionally, activity decreases first in the putamen and then in the caudate nucleus, as indicated by neurologic signs (11). Thus, $\mathrm{ROI}_{\max }$ is not considered appropriate for evaluating the activity of the entire striatum. In view of these considerations, $\mathrm{ROI}_{\mathrm{CT} 2}$ was most appropriate for analyzing ${ }^{123} \mathrm{I}-\mathrm{FP}-\mathrm{CIT}$ SPECT in this study. $\mathrm{ROI}_{\mathrm{SP} 2}$ and $\mathrm{ROI}_{\mathrm{SP} 3}$ showed lower $\mathrm{SBR}_{\mathrm{SPECT}}$. Delineation of the striatum on SPECT images was difficult, and the delineated area was usually larger than the anatomic contour. Bolt et al. automatically placed large pentagonal ROIs to cover the entire striatum and measured the total striatal count (21). They obtained the striatal concentration by dividing the total striatal counts by a fixed striatal volume of $11.2 \mathrm{~cm}^{3}$. Although they obtained good results using this method, the striatal volume is likely to be different from that of patients with neurologic disorders. ROI $_{\mathrm{CT} 2}$ and $\mathrm{ROI}_{\mathrm{CT} 3}$ in our study were determined on individual CT images - an advantage of using a SPECT/CT hybrid device.

More precisely, the activity of the putamen and caudate nucleus should be evaluated separately. A decrease in putaminal

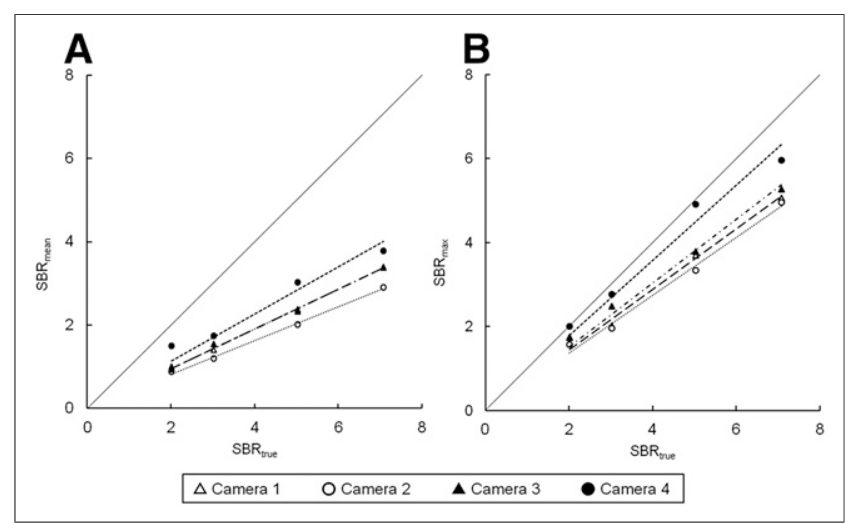

FIGURE 4. Correlation of $\mathrm{SBR}_{\text {true }}$ and $\mathrm{SBR}_{\text {SPECT }}$ of $\mathrm{SBR}_{\text {mean }}$ (A) and $\mathrm{SBR}_{\max }$ (B). Highest recovery was obtained by camera 4 for both $\mathrm{SBR}_{\text {mean }}$ and $\mathrm{SBR}_{\text {max }}$. 
TABLE 6

Linearity and Recovery of SBR SPECT $_{\text {for Different Devices and Collimators }}$

\begin{tabular}{|c|c|c|c|c|c|c|}
\hline \multirow[b]{2}{*}{ Parameter } & \multicolumn{4}{|c|}{$\mathrm{SBR}_{\text {true }}$} & \multirow[b]{2}{*}{ Average } & \multirow[b]{2}{*}{ Linearity $R^{2}$} \\
\hline & 2.01 & 3.03 & 5.03 & 7.08 & & \\
\hline \multicolumn{7}{|l|}{$\mathrm{SBR}_{\text {mean }}$} \\
\hline Camera 1 & $49.3 \%$ & $46.5 \%$ & $47.3 \%$ & $47.8 \%$ & $47.7 \% \pm 1.0 \%$ & 1.00 \\
\hline Camera 2 & $43.9 \%$ & $39.2 \%$ & $39.9 \%$ & $41.0 \%$ & $41.0 \% \pm 1.8 \%$ & 1.00 \\
\hline Camera 3 & $46.8 \%$ & $51.0 \%$ & $46.4 \%$ & $47.9 \%$ & $48.0 \% \pm 1.8 \%$ & 1.00 \\
\hline Camera 4 & $67.4 \%$ & $54.4 \%$ & $66.4 \%$ & $58.3 \%$ & $61.6 \% \pm 5.5 \% *$ & 0.97 \\
\hline \multicolumn{7}{|l|}{$\mathrm{SBR}_{\max }$} \\
\hline Camera 1 & $86.0 \%$ & $66.4 \%$ & $73.2 \%$ & $71.4 \%$ & $74.3 \% \pm 7.2 \%$ & 0.98 \\
\hline Camera 2 & $77.9 \%$ & $64.3 \%$ & $66.2 \%$ & $70.0 \%$ & $59.6 \% \pm 5.2 \%$ & 0.99 \\
\hline Camera 3 & $86.4 \%$ & $81.6 \%$ & $75.0 \%$ & $74.3 \%$ & $79.3 \% \pm 5.0 \%$ & 0.99 \\
\hline Camera 4 & $99.2 \%$ & $90.8 \%$ & $97.4 \%$ & $84.2 \%$ & $92.9 \% \pm 5.9 \% *$ & 0.97 \\
\hline${ }^{\star} P<0.05$ & & & & & & \\
\hline
\end{tabular}

uptake without a decrease in caudate uptake is observed in the early stage of Parkinson disease (1). Some papers have reported the usefulness of the putamen-to-caudate ratio for differential diagnosis using separately placed ROIs on the putamen and caudate nucleus $(22,23)$. Furthermore, the putamen may also need to be analyzed by being divided into different parts for some disorders (24). The usefulness of statistical parametric mapping, in comparison with ROI analysis, for evaluating striatal uptake has been reported $(25,26)$. Stereotactic normalization and standardized comparisons are expected to allow more reliable evaluation. Further analyses using statistical image analysis of striatal uptake with detailed information about the striatal structure may be required.

In this study, $\mathrm{SBR}_{\text {mean }}$ was never more than $67.4 \%$ of $\mathrm{SBR}_{\text {true }}$. Small structures such as the striatum are associated with an underestimation of radioactivity because of the partial-volume effect $(27,28)$, which is attributable to the limited spatial resolution of SPECT. The radioactivity concentration of the striatum was underestimated by more than $50 \%$ without partial-volume correction in a previous study (29). Soret et al. reported that partial-volume correction was especially effective for reducing putaminal uptake (30). Bolt et al. suggested that a 3D ROI is more appropriate than a 2D ROI for considering partial-volume effect (21). In our study, 4 ROIs were used for analyses on a single slice (2D), and 3 ROIs on 5 slices were used for averaging (3D). Although the results did not significantly differ between 2D and 3D ROIs, evaluation of striatal uptake should ideally be performed for 3D ROIs in future studies because there were some differences.

The primary $\gamma$ ray of ${ }^{123} \mathrm{I}$ has energy of $159 \mathrm{keV}(83.3 \%)$, although several other $\gamma$ rays of higher energy, ranging from 248 to $784 \mathrm{keV}$, are also emitted (31). These highenergy $\gamma$ rays and scattered photons penetrate the thin septa of the collimator. From this point of view, the use of a collimator designed for higher-energy $\gamma$ rays may be recommended. Even though both camera 1 and camera 3 were equipped with collimators for low- and medium-energy $\gamma$ rays, recovery of $\mathrm{SBR}_{\text {mean }}$ was second best for camera 3 and third best for camera 1. Furthermore, the striatal uptake was broad and rounded for both camera 1 and camera 3 . The spatial resolution of low- to medium-energy collimators is inferior to that of a high-resolution collimator. Thus, SBR is considered to be underestimated because of the partialvolume effect. Camera 2, equipped with a low-energy collimator, showed the lowest recovery of $\mathrm{SBR}_{\text {mean }}$ and poor image quality. Because the characteristics of low-energy collimators vary among vendors, the thin septa and short hole length of camera 2 deteriorated the image quality of ${ }^{123}$ I (32). Although the guidelines for ${ }^{123}$ I-FP-CIT SPECT recommend the use of a LEHR collimator because of the benefit of higher spatial resolution $(5,6)$, the collimator used for camera 2 should not be used for imaging ${ }^{123}$ I-FP-CIT. On the other hand, the best SBR and the best image quality were obtained by camera 4 in this study. Although both camera 2 and camera 4 used LEHR collimators, the configuration and basic characteristics of the collimators were different. These results suggest that the LEHR collimator of camera 4 can be used for ${ }^{123}$ I-FP-CIT imaging.

This study had several limitations. First, we did not examine the fanbeam collimator. Use of a fanbeam collimator for ${ }^{123} \mathrm{I}$ is expected to provide better results because of its high sensitivity and high spatial resolution. Second, SPECT images were reconstructed by filtered backprojection from Chang attenuation correction because it has been used as a standard quantitative method for brain SPECT in our country. Recent advances in iterative reconstruction have improved both image quality and quantitative accuracy. The use of iterative reconstruction should be examined in future studies. Third, attenuation correction was performed using the Chang method. Although CT-based attenuation correction can be used in our institution, it has not yet become widely available in most clinical institutions. CT-based attenuation correction should be examined in future studies. Finally, several other correction methods, such as the spatial resolution correction, should also be examined in the future. 


\section{CONCLUSION}

Our results suggest that the ROI contouring of the striatum based on CT images was appropriate for evaluating DaT SPECT images. Among the different SPECT/CT devices, the best $\mathrm{SBR}_{\text {mean }}$ was obtained with camera 4 equipped with an LEHR collimator designed to be used for ${ }^{123}$ I imaging.

\section{DISCLOSURE}

No potential conflict of interest relevant to this article was reported.

\section{REFERENCES}

1. Djang DSW, Janssen MJR, Bohnen N, et al. SNM practice guideline for dopamine transporter imaging with ${ }^{123}$ I-ioflupane SPECT 1.0. J Nucl Med. 2012;53:154-163.

2. Darcourt J, Booij J, Tatsch K, et al. EANM procedure guidelines for brain neurotransmission SPECT using ${ }^{123}$ I-labelled dopamine transporter ligands, version 2. Eur J Nucl Med Mol Imaging. 2010;37:443-450.

3. O'Sullivan JD, Lees AJ. Nonparkinsonian tremors. Clin Neuropharmacol. 2000;23:233-238.

4. Furukawa Y, Kish SJ. Dopa-responsive dystonia: recent advances and remaining issues to be addressed. Mov Disord. 1999;14:709-715.

5. Albanese A, Colosimo C, Lees AJ, Tonali P. The clinical diagnosis of multiple system atrophy presenting as pure parkinsonism. Adv Neurol. 1996;69:393-398.

6. Catafau AM, Tolosa E. Impact of dopamine transporter SPECT using ${ }^{123}$ Iioflupane on diagnosis and management of patients with clinically uncertain parkinsonian syndromes. Mov Disord. 2004;19:1175-1182.

7. Booij J, Tissingh G, Boer GJ, et al. ${ }^{[123}$ I]FP-CIT SPECT shows a pronounced decline of striatal dopamine transporter labelling in early and advanced Parkinson's disease. J Neurol Neurosurg Psychiatry. 1997;62:133-140.

8. Tissingh G, Bergmans P, Booji J, Winogrodzka A, Stoof JC, van Royen EA $\left[{ }^{123} I\right] \beta$-CIT single-photon emission tomography in Parkinson's disease reveals a smaller decline in dopamine transporter s with age than in controls. Eur J Nucl Med. 1997;24:1171-1174.

9. Lavalaye J, Booji J, Reneman L, Habraken JB, van Royen EA. Effect of age and gender on dopamine transporter imaging with $\left[{ }^{123} \mathrm{I}\right]$-FP-CIT SPET in healthy volunteers. Eur J Nucl Med. 2000;27:867-869.

10. Badiavas K, Molyvda E, Iakovou I, Tsolaki M, Psarrakos K, Karatzas N. SPECT imaging evaluation in movement disorders: far beyond visual assessment. Eur J Nucl Med Mol Imaging. 2011;38:764-773.

11. Tissingh G, Booij J, Bergmans P, et al. Iodine-123-N- $\omega$-fluoropropyl-2 $\beta$-carbomethoxy$3 \beta$-(4-iodophenyl)tropane SPECT in healthy controls and early-stage, drug-naive Parkinson's disease. J Nucl Med. 1998;39:1143-1148.

12. Seibyl JP, Marek K, Sheff K, et al. Iodine-123- $\beta$-CIT and iodine-123-FPCIT SPECT measurement of dopamine transporters in healthy subjects and Parkinson's patients. J Nucl Med. 1998;39:1500-1508.

13. Booij J, Habraken JB, Bergmans P, et al. Imaging of dopamine transporters with iodine-123-FP-CIT SPECT in healthy controls and patients with Parkinson's disease. J Nucl Med. 1998;39:1879-1884

14. Chouker M, Tatsch K, Linke R, Progarell O, Hahn K, Sckwarz J. Striatal dopamine transporter binding in early to moderately advanced Parkinson's disease: monitoring of disease progression over 2 years. Nucl Med Commun. 2001;22:721-725.
15. Pirker W, Djamshidian S, Asembaum S, et al. Progression of dopaminergic degeneration in Parkinson's disease and atypical parkinsonism: a longitudinal betaCIT SPECT study. Mov Disord. 2002;17:45-53.

16. Yoneda H, Shirao S, Koizumi H, et al. Reproducibility of cerebral blood flow assessment using a quantitative SPECT reconstruction program and split-dose ${ }^{123}$ I-iodoamphetamine in institutions with different $\gamma$-cameras and collimators. J Cereb Blood Flow Metab. 2012;32:1757-1764

17. Chang LT. A method for attenuation correction in radionuclide computed tomography. IEEE Trans Nucl Sci. 1978;25:638-642.

18. Iida $\mathrm{H}$, Hori $\mathrm{Y}$, Ishida $\mathrm{K}$, et al. Three-dimensional brain phantom containing bone and grey matter structures with a realistic head contour. Ann Nucl Med. 2013;27: 25-36.

19. Akamatsu M, Yamashita Y, Akamatsu G, et al. Influences of reconstruction and attenuation correction in brain SPECT images obtained by the hybrid SPECT/CT device: evaluation with a 3-dimensional brain phantom. Asia Oceania J Nucl Med Biol. 2014;2:24-29.

20. Gerasimou G, Costa DC, Pspsnastasiou E, et al. SPECT study with I-123-ioflupane (DaTSCAN) in patients with essential tremor: is there any correlation with Parkinson's disease? Ann Nucl Med. 2012;26:337-344.

21. Tossici-Bolt L, Hoffman SMA, Kemp PM, Mehta RL, Fleming JS. Quantification of ${ }^{123}$ I-FP-[CIT] SPECT brain images: an accurate technique for measurement of the specific binding ratio. Eur J Nucl Med Mol Imaging. 2006;33:1491-1499.

22. Lorberboym M, Djaldetti R, Melamed E, Sadeh M, Lampl Y. ${ }^{123}$ I-FP-CIT SPECT imaging of dopamine transporters in patients with cerebrovascular disease and clinical diagnosis of vascular parkinsonism. J Nucl Med. 2004;45:1688-1693.

23. Løkkegaard A, Werdelin LM, Friberg L. Clinical impact of diagnostic SPET investigations with a dopamine re-uptake ligand. Eur J Nucl Med. 2012;29:1623-1629.

24. Braga-Neto P, Felicio AC, Hoexter MQ, et al. Cognitive and olfactory deficits in Machado-Joseph disease: a dopamine transporter study. Parkinsonism Relat Disord. 2012;18:854-858.

25. Weeks RA, Cunningham VJ, Piccini P, Waters S, Harding AE, Brooks DJ. ${ }^{11} \mathrm{C}$ diprenorphine binding in Huntington's disease: a comparison of region of interest analysis with statistical parametric mapping. J Cereb Blood Flow Metab. 1997; 17: 943-949.

26. Kas A, Payoux P, Habert M, et al. Validation of a standardized normalization template for statistical parametric mapping analysis of ${ }^{123}$ I-FP-CIT images. J Nucl Med. 2007;48:1459-1467.

27. Frouin V, Comtat C, Reilhac A, Grégoire M. Correction of partial-volume effect for PET striatal imaging: fast implementation and study of robustness. $\mathrm{J} \mathrm{Nucl}$ Med. 2002;43:1715-1726.

28. Soret M, Koulibaly PM, Darcourt J, Hapdey S, Buvat I. Quantitative accuracy of dopaminergic neurotransmission imaging with ${ }^{123}$ I SPECT. J Nucl Med. 2003; 44:1184-1193.

29. Buvat I, Soret M, Hapdey S, Riddell C, Benali H, Di Paola R. Respective importance of scatter, attenuation, collimator response and partial volume effect corrections for accurate quantification in ${ }^{123} \mathrm{I}$ dopamine receptor imaging. In: 2001 IEEE Nuclear Science Symposium and Medical Imaging Conference [CDROM]. Piscataway NJ: IEEE; 2000:13-15-13-19.

30. Soret M, Koulibaly PM, Darcourt J, Buvat I. Partial volume effect correction in SPECT for striatal uptake measurements in patients with neurodegenerative disease: impact upon patient classification. Eur J Nucl Med Mol Imaging. 2006;33:1062-1072.

31. Snay ER, Treves T, Fahey FH. Improved quality of pediatric ${ }^{123}$ I-MIBG images with medium-energy collimators. J Nucl Med Technol. 2011;39:100-104.

32. He X, Frey EC, Links JM, Song X, Tsui BMW. Comparison of penetration and scatter effects on defect contrast for GE and Siemens LEHR collimator in myocardial perfusion SPECT: a simulation study. IEEE Trans Nucl Sci. 2005;52:1359-1364. 